\title{
Actualización en crioterapia. Usos en ginecología
}

\author{
Dr. Gildardo Gallego Noreña*
}

RESUMEN. Este artículo presenta una información global acerca de la crioterapia.

La introducción hace énfasis en que la terapia usual para NIC (neoplasia intraepitelial cervical) era la cirugía y hoy la crioterapia es una alternativa conservadora para mujeres jóvenes que quieren preservar la vida reproductiva.

Presenta además aspectos históricos y luego explica el mecanismo de acción, dispositivo técnico, usos generales en medicina y ginecología.

Los usos en el campo de la ginecología son muy importantes en entidades como cervicitis benigna, NIC, leucoplasias cervicales, papiloma virus humano y herpes genital.

La orientación general va dirigida a los aspectos de tratamiento del NIC III. Las pacientes con NIC III deberán tener como requisitos mínimos: La lesión debe estar confinada estrictamente al cuello, el diámetro debe ser menor de tres centímetros y las márgenes de congelación deben pasar los cuatro milímetros lejos de la lesión.

La paciente no debe tener coitos a usar tampones los siguientes diez días.

Esta modalidad terapéutica se usó para pacientes con papiloma virus asociado o no con neoplasia, es una alternativa para mujeres embarazadas que cursan el primero y segundo trimestre.

Finalmente muestra diagramas del criocauterio y se cita la bibliografía más relievante consultada.

PALABRAS CLAVES: Crioterapia, NIC (neoplasia cervical intrapietelial), papiloma virus humano.

SUMMARY. This paper presents a global information about cryotherapy.

The introduction make emphasis that the usual therapy for NIC (cervical intraephitelial neoplasia) was the surgery in other time and today the cryotherapy is an conservative alternative for young woman that likes preserve reproductive life.

It presents beside the historics aspects and explain the action mecanism, tecnical device, uses en general medicine and gynecology.

The utilities in the camp of gynecology are very important in entities as: benign cervicitis, NIC, cervical and vulvar leukoplaquia, human papillomavirus polips and genital herpes.

The general orientation go to gynecologis aspects about therapy of NIC III.

The patients with NIC III.

Would have minim requisit as, the lesion be confined at the cervical area, and the diameter of this area would be minus three centimeters, and margin of congelation will be four $\mathrm{mm}$ far lesion.

The patient will be have no coitus or use tampons for next ten days after therapy.

This modalities of therapy was used for patients with human papilloma virus asociated or no with neoplasia, is an alternative for pregnancy woman that courses first and second trimester.

Finally shows diagrams as model of device and the bibliografy more important of about this teme.

KEY WORDS: Cryotherapy, NIC (cervical intraepithelial neoplasia), human papilloma virus.

\section{Introducción}

Con el avance tecnológico de la colposcopia para la evaluación del tracto genital femenino (vulva-vagina y cervix) de pacientes con citologías anormales, muchos ginecólogos han cambiado substancialmente su mentalidad quirúrgica por tratamientos más conservadores, menos mutilantes y que se puedan realizar ambulatoriamente.

La conización y la histerectomía han constituido tradicionalmente el tratamiento rutinario para el NIC (neoplasia intraepitelial cervical) dependiendo de la edad y del futuro obstétrico; estos procedimientos necesariamente requieren

* Profesor Oncología Ginecológica. Universidad de Antioquia, Facultad de Medicina, Departamento de Obstetricia y Ginecología. hospitalización, anestesia regional o general y las complicaciones que usualmente se presentan han variado entre un inco y diez por ciento.

Desde hace unas décadas en adelante se habla de modalidades terapéuticas conservadoras, éstas son: la electrocoagulación, la coagulación con rayos infrarrojos, la vaporización con rayos láser de $\mathrm{CO}_{2}$, la cual es exageradamente costosa, y la crioterapía.

$\mathrm{Al}$ realizar tratamientos conservadores con métodos destructivos locales, debemos recordar que no se puede obtener una muestra o espécimen para estudio histopatológico postoperatorio, lo cual exige por parte del médico una completa seguridad del diagnóstico preoperatorio, pues de lo contrario se puede estar enmascarando un carcinoma microinvasor o francamente invasor. 
La crioterapia es actualmente muy usada en el campo médico de la otorrino, oftalmología, proctología y en el campo ginecológico que es el aspecto del cual hablaremos.

\section{Aspectos históricos}

Desde hace unos 23 años se ha usado la crioterapia en el campo ginecológico, pero su historia data desde 1883 cuando Openchowski citado por Elsworth (10) usó corrientes de agua helada para tratar algunas lesiones ginecológicas. En 1940 Weitzner utilizó hielo seco para tratar cervicitis crónica, y en 1967 Crisp usó la crioterapia como tratamiento del NIC (10).

\section{Principio básico}

La crioterapia es la congelación de tejidos mediantedispositivos técnicos especiales con los cuales se produce necrosis.

Existen dos mecanismos físicos de congelación:

1. Cambio de fase: Es la evaporación de un líquido o sólido en el cual el gas circula a través de una probeta.

2. Expansión isotrópica adiabática de compresión de gas (efecto Joule Thompson) o sea la expansión rápida de un gas a través de un orificio estrecho induce un descenso en la temperatura, las presiones pueden variar entre 750 y 900 libras por pulgada cuadrada y el diámetro del orificio varía entre 0.013 y 0.018 pulgadas (10). La temperatura dentro de la boquilla de la sonda es igual al punto de ebullición del gas que sea utilizado.

La temperatura varía de acuerdo con el gas que se use: óxido nitroso (-89 grados centígrados), dióxido de carbono (-78 grados centígrados). El metal de la boquilla puede ser de oro, plata o cobre. La temperatura de congelación ideal debe ser menor de 60 grados centígrados.

La técnica de fa crioterapia es muy simple, pero se deben tener presentes algunos requisitos para lograr una congelación ideal, el gas que se use: óxido nitroso o dióxido de carbono, debe usarse refrigerado. Se debe preferir el tanque grande a los tanques angostos, especialmente si se realiza criocirugía a varias pacientes en un período corto de tiempo, porque la presión en el tanque pequeño cae rápidamente aunque este tenga suficiente gas; si no hay buena presión no se logra buena congelación. Se aconseja una capa delgada de lubricante soluble en agua sobre la boquilla para permitir una rápida y uniforme congelación del cervix.

Los aparatos técnicos son muy variados en el comercio de acuerdo con diferentes marcas, pero el principio fundamental es el mismo.

Una copa de adaptación al cervix, una pistola para descongelar, una válvula de escape de gas y un tanque con gas (ver dibujo No. 1).

La congelación del tejido se logra de tres maneras:

1. Técnica de inserción: Colocando la sonda intratumoral.

2. Técnica de contacto: Aplicando la boquilla sobre el tejido lesionado y es la más usada en el mundo.

3. Congelación tipo aerosol de nitrógeno líquido (ver dibujo No. 2).

Esquemas terapéuticos de congelación:

Se han utilizado dos esquemas terapéuticos para realizar la crioterapia cervical:
Dibujo No.1

CRIOCAUTERIO

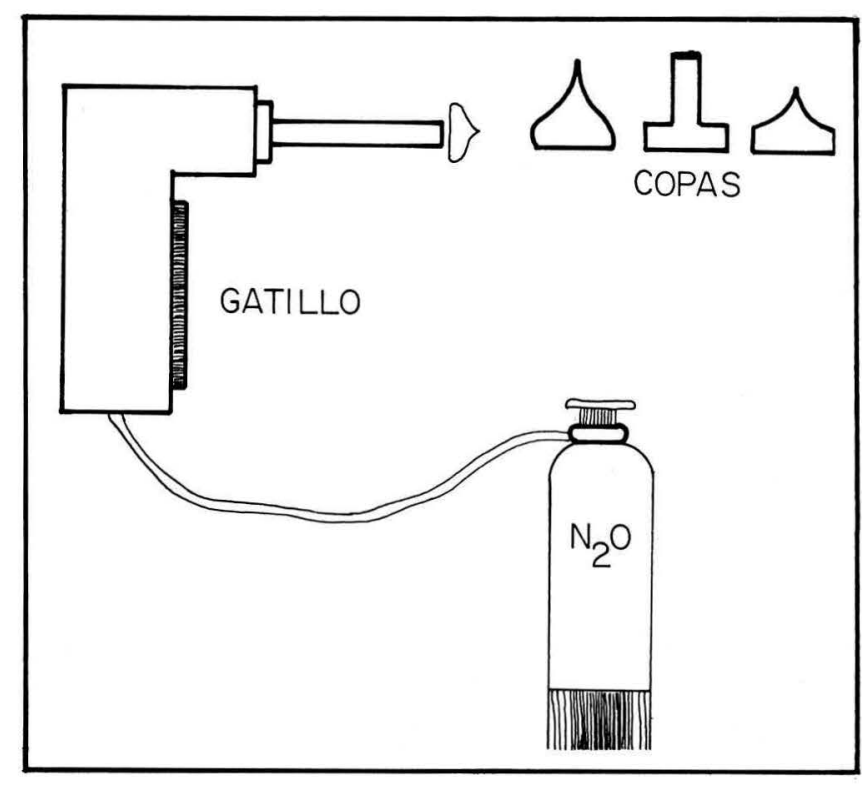

Dibujo No.2

TECNICAS DE CONGELACION TISULAR

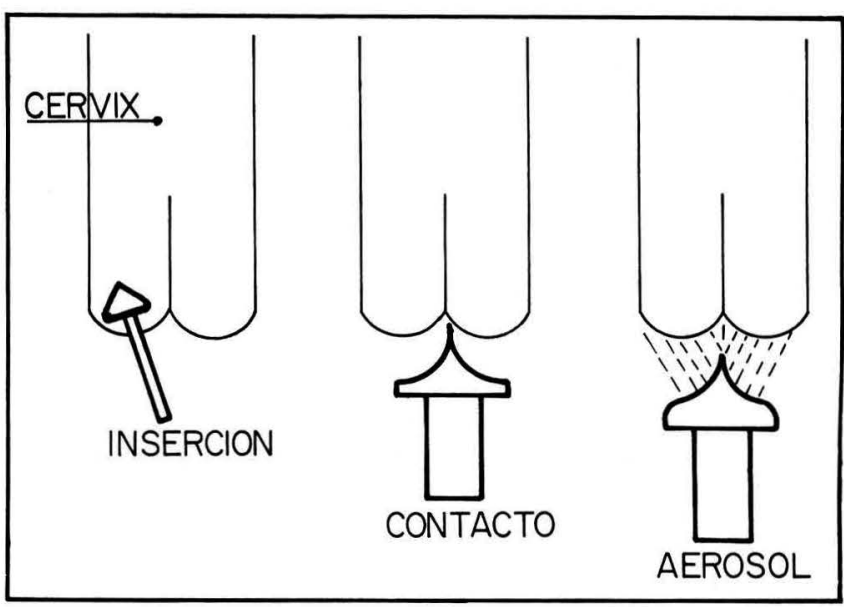

1. Congelación simple: Con una duración de congelación de tres a cinco minutos.

2. Doble congelación: Tres minutos de congelación, cinco de descanso y termina con otros tres de congelación (6-9). Con esta técnica se han obtenido los mejores resultados (8, 9).

Mecanismo de acción:

Los tejidos sometidos a crioterapia experimentan cambios anatomofisiológicos que debemos tener en cuenta para la evaluación cervical de estas pacientes. Estos cambios son: 1. Deshidratación y concentración tóxica de electrolitos debido a rẹmoción de agua.

2. Cristalización con ruptura de la membrana basal.

3. Desnaturalización de proteínas dentro de la membrana celular.

4. Choque térmico.

5. Estasis vascular (10). 
Estos mecanismos causan necrosis del tejido que es eliminado.

La evaluación microscópica del cuello post-tratamiento experimenta cambios que se pueden demostrar según Kaufman citado por Elsworth (10): Este autor con biopsias seriadas encontró a las 24 horas necrosis extensa y no observó epitelio, dos semanas más tarde halló tejido de granulación, numerosos capilares, proliferación fibroblástica, infiltración leucocitaria y epitelio de regeneración. Al mes el epitelio es escaso, inmaduro y a las ocho semanas (dos meses) el tejido del cuello es normal.

La nueva unión escamocolumnar se coloca justo dentro del canal, lo cual es importante para el seguimiento citológico y colposcópico.

Al microscopio electrónico en el epitelio cervical se observan inmediatamente después de la crioterapia, edema mitocondrial y ruptura de su capa interna, destrucción de cristales tanto en el tejido epitelial como en el tejido conectivo. El daño mitocondrial más severo se presentó en las células basales del epitelio, en fibroblastos y en capilares del tejido conectivo. El daño fue menor en células parabasales y en células intermedias; a menudo se fragmentan las membranas y se condensa el núcleo (22).

\section{Indicaciones ginecológicas de la crioterapia}

Los usos de la crioterapia en el campo ginecológico son amplios, incluyen lesiones vulvares, vaginales y cervicales. Las lesiones que se pueden tratar con crioterapia son:

1. Cervicitis crónica benigna.

2. Displasias y NIC (en sus diversos grados).

3. Leucoplasias cervicales y vulvares.

4. Controlar hemorragia postconización.

5. Condilomas acuminados vulvares, vaginales y cervicales.

6. Quistes de Naboth.

7. Pólipos.

8. Herpes genital.

Desde este punto de vista ginecológico haré más énfasis en el tratamiento del NIC, ya que esta entidad se diagnostica cada vez con más frecuencia, el número de pacientes jóvenes con esta entidad aumenta y muchas de estas no han tenido hijos. Además, si tratamos la neoplasia intraepitelial temprano y exitosamente, estamos disminuyendo la morbimortalidad de la mujer al impedir que la lesión progrese al carcinoma invasor.

\section{Recomendaciones}

Para realizar la crioterapia y obtener resultados se recomienda:

1. Un cuidadoso estudio del cuello uterino, el cual debe incluir: visión completa de la unión escamocolumnar para determinar la extensión de la lesión; no debe existir lesión en los criptas glandulares o en el canal endocervical; si existe alguna duda se debe realizar curetaje endocervical y de acuerdo con este se recomienda tomar la conducta; cuando el resultado del curetaje endocervical es positivo lo ideal es realizar una conización diagnóstica.

2. No debe existir lesión en fondos de saco vaginales o paredes de la misma, pues la crioterapia no cubre lesiones $\tan$ extensas.

3. El área de la lesión debe ser menor de tres centímetros de diámetro.

4. De efectuar la crioterapia se prefiere que el margen de congelación llegue a cuatro milímetros por fuera de la lesión. 5. Se debe advertir a la paciente la presencia de hidrorrea y leucorrea por un período de cuatro semanas.

6. La paciente no debe usar tampones y abstenerse de relaciones sexuales durante este período.

La efectividad de la crioterapia expresada en términos de años de seguimiento es importante considerarla porque los porcentajes de curación pueden ser sesgados si el seguimiento es corto. Consultada la literatura mundial el seguimiento más largo es el informado por Benedet (6) de diez años, Hemmingsson (16) ocho años y Peckhman (24) cuatro años.

El éxito del tratamiento depende de la extensión de la lesión y de los cuadrantes afectados $(1,8,12,19)$.

Arof (1) informa un 84 por ciento de éxito promedio de curación, él considera que el área de la lesión por cuadrante es de gran importancia para un buen resultado terapéutico (ver dibujo No. 3).

Dibujo No.3

DIAGRAMA CERVICAL

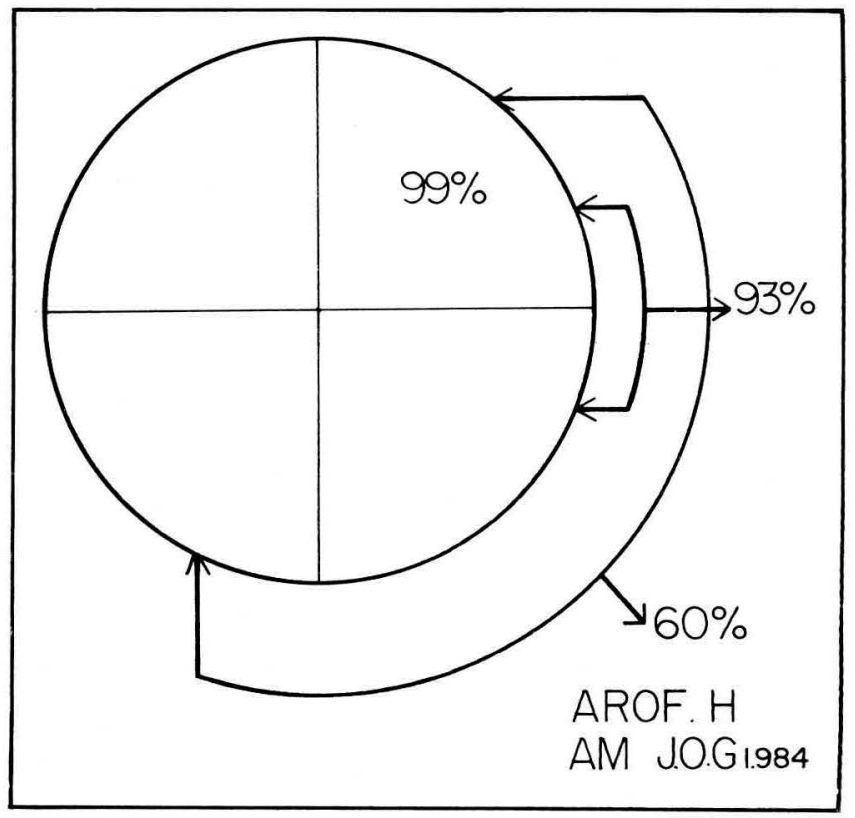

Cuando sólo existe un cuadrante cervical comprometido el éxito de curación es de 99 por ciento. Si el área de la lesión compromete dos cuadrantes, el éxito es de 93 por ciento y si la lesión compromete tres o los cuatro cuadrantes el éxito de curación baja al 60 por ciento. Este cuadro es muy ilustrativo para tratar de seleccionar las pacientes candidatas a crioterapia con lesiones exclusivamente pequeñas.

También este mismo autor encontró una correlación muy significativa entre el área de la lesión y la severidad histológica; a medida que el NIC progresa en gravedad histológica el área topográfica de la lesión tendió a aumentar según se puede apreciar en el Cuadro 1 tomado de Arof H. (1).

En un sentido general se puede decir que el éxito del tratamiento no depende del método mismo, sino de un buen 
Cuadro 1

EXITO DE CRIOTERAPIA VS. GRADO HISTOLOGICO DEL NIC

\begin{tabular}{|cccc|}
\hline Tipo & Númerototal de & \multicolumn{2}{c|}{ Número pacientes curadas } \\
histológico & pacientes tratadas & No. & $\%$ \\
\hline NICI & 120 & 110 & 91 \\
NIC II & 92 & 75 & 81 \\
NIC III & 53 & 40 & 72 \\
\hline
\end{tabular}

protocolo de investigación en el cual se efectúe una adecuada evaluación pretratamiento, una selección estricta de las pacientes y un excelente seguimiento, lo que necesariamente evitaría fracasos terapéuticos (2).

\section{Justificación de la crioterapia como trata- miento del NIC}

En Colombia la primera causa de mortalidad en mujeres entre los 15 y 44 años es el carcinoma de cuello. El diagnóstico del carcinoma invasor lentamente va disminuyendo y el carcinoma in situ o NIC va en aumento debido a un mejor diagnóstico. En 1980 la incidencia de cáncer cervical era de 88 casos nuevos por 100.000 habitantes (3). Con el cambio de las costumbres sexuales se diagnostica cada vez más temprano neoplasias cervicales en adolescentes.

Jones D. (17) encontró en un estudio de 311 adolescentes con citologías anormales que el 42 por ciento tienen NIC I, el 22 por ciento NIC II y el 22 por ciento NIC III, todas las lesiones estaban en el exocervix y sólo un tres por ciento se encontraba en endocervix, lo que obligó a realizar cono diagnóstico.

Se ha postulado que algo ha cambiado en la historia natural del cáncer cervical, puesto que el diagnóstico se efectúa en pacientes cada vez más jóvenes, pero no se ha encontrado un factor etiológico especial, por el contrario Zaninitti P. (32) realiza un estudio de 126 casos y 1.914 controles en mujeres por debajo de 20 años en los cuales encuentra una alta correlación del inicio de relaciones sexuales precoces, promiscuidad y lesiones verrugosas por papiloma virus con el NIC y ninguna asociación en los controles. Estas características ameritan que se investigue más sobre tratamientos conservadores tipo crioterapia para pacientes adolescentes que aún no han desarrollado la etapa reproductiva y están en plena actividad sexual.

En el Hospital San Vicente de Paúl de Medellín, Gallego y Uribe (14) estudiaron 1.276 casos de NIC III en un período de 17 años (1970-1987) y encontraron que un gran porcentaje de pacientes eran muy jóvenes, el 18.7 por ciento estaban entre 20 y 29 años, el 38.5 por ciento entre 30 y 39 años y el 25 por ciento entre 40 y 49 años; en estos pacientes el tratamiento fue exclusivamente quirúrgico así: cono en el 22.7 por ciento, histerectomía en el 68 por ciento y cono e histerectomía en el dos por ciento; no hubo tratamientos conservadores a pesar de que la gran mayoría de pacientes estaban en edad.reproductiva.

La crioterapia se impone como una forma conservadora de tratamiento para pacientes con NIC y con otras lesiones cervicales, vaginales y vulvares. Como enunciamos anteriormente, desde hace dos décadas y media se ha utilizado la crioterapia como tratamiento del NIC en sus tres estadios con éxitos en el tratamiento que fluctúan entre el 60 y 95 por ciento $(4,5,11,12,18,19,27,28)$. Varios factores se deben tener presentes para explicar esta variedad de resultados, estos son: experiencia del operador, número de pacientes tratadas, criterios establecidos para determinar curación, años de seguimiento, técnica de congelación, equipo y gas usado (9).

El Cuadro 2 ilustra la comparación del láser y la crioterapia de acuerdo con varios estudios, los porcentajes de curación son similares para los dos métodos y las complicaciones según los autores son muy insignificantes, la disponibilidad de la crioterapia y los costos más favorables lo hacen el método de elección.

Cuadro 2

ESTUDIOS COMPARATIVOS DE CRIOTERAPIA VS. LASER

\begin{tabular}{|c|c|c|c|c|c|}
\hline Autor & Tipo de estudio & $\begin{array}{l}\text { Crioterapi } \\
\text { Número } \\
\text { pacientes }\end{array}$ & $\%$ & \begin{tabular}{l}
\multicolumn{1}{c}{ Láser } \\
Número \\
pacientes
\end{tabular} & $\%$ \\
\hline Towsend D. (28) & Comparativo & 100 & 93 & 100 & 89 \\
\hline Kwikkel(19) & Al azar & 50 & 86 & 51 & 71 \\
\hline Kirwan (18) & $\begin{array}{l}\text { Prospectivo } \\
\text { Comparativo }\end{array}$ & 35 & 94 & 71 & 97 \\
\hline Berget (4) & $\begin{array}{l}\text { Prospectivo } \\
\text { Al azar }\end{array}$ & 101 & 85 & 103 & 91 \\
\hline Van Lent (34) & $\begin{array}{l}\text { Descriptivo } \\
\text { Prospectivo }\end{array}$ & 153 & 90 & & \\
\hline
\end{tabular}

Por todas las razones expuestas, considero necesario adquirir experiencia con esta modalidad terapéutica.

A nivel nacional un estudio realizado en la Universidad Javeriana de Santafé de Bogotá, Perdomo R. (23) trata 30 pacientes con NIC de las cuales doce tenían NIC I, diez NIC II y ocho NIC III. Se utilizó la técnica de doble congelación. En este estudio no se informa sobre el seguimiento, dato básico para evaluar la efectividad del tratamiento.

A rivel local se realiza un estudio comparativo entre crioterapia y tratamiento quirúrgico (cono e histerectomía) para el NIC III. Se han evaluado 432 pacientes de las cuales 132 fueron tratadas con crioterapia y 300 con cirugía, se concluye que la persistencia con los dos tratamientos son similares, recurrencias aún no se h́an presentado, pero el seguimiento aún es corto, la investigación continúa.

\section{NIC y papiloma virus}

La crioterapia actualmente ocupa un lugar importante en el tratamiento ambulatorio de pacientes con neoplasia intraepitelial cervical y de lesiones condilomatosas tanto de cuello uterino como de vagina y vulva.

Hay evidencia de la asociación de papiloma virus humano (HPV) con la neoplasia intraepitelial cervical y también una clara relación entre el papiloma y las relaciones sexuales. Se ha informado de la presencia de ADN del papiloma virus en el 90 por ciento de los cánceres cervicales tanto in situ como invasores.

Los serotipos relacionados directamente con el cáncer cervical (NIC) son el 16 y 18 y últimamente se han relacionado los 11 y 31 aunque con una actividad oncogénica menor que los anteriores (29). 
La historia natural del NIC es similar a las infecciones por papiloma virus, sólo que estos últimos ocurren en pacientes por lo menos diez años menores que en aquellas que desarrollan NIC (26), esto puede indicar que el papiloma acelera la neoplasia intracervical. Los efectos citopáticos de coilocitosis causados por el papiloma virus, se observan tistológicamente en forma idéntica tanto en los cándilomas como en algunos grados de NIC. Desafortunadamente no existe en Colombia la tecnología para tipificar los serotipos del papiloma y poder determinar cuáles son los verdaderamente oncogénicos y así tratarlos. Se ha sugerido no tratar y sólo observar a la colposcopia, pero creemos que esto causaría gran ansiedad a las pacientes por tratarse de una infección de fácil transmisión. Los tratamientos recomendados para eliminar la lesión son la electrocauterización y la crioterapia; no parece haber diferencia a la respuesta al tratamiento de los NIC asociados con condiloma o no.

También la crioterapia se ha usado para pacientes con NIC III durante el embarazo, este procedimiento ofrece menos complicaciones que los métodos quirúrgicos tradicionales. El sangrado, la necrosis y la infección secundaria son menores después de crioterapia que con otras técnicas.

Se ha usado en los tres trimestres del embarazo, pero en el tercer trimestre se han descrito algunas complicaciones como el parto pretérmino por ruptura de membranas (15, 21). Además, el paso del feto por el canal del parto en pacientes con infección viral puede producir contaminación del papiloma en la laringe del niño, por lo tanto la indicación de la crioterapia es evidente en las pacientes embarazadas con infección viral.

También se recomienda una búsqueda de contactos, además, excluir otras enfermedades sexualmente transmisibles como parte integral del manejo de condilomas y verrugas genitales (31).

\section{Complicaciones}

Las complicaciones que se han descrito post-crioterapia son muy escasas, algunos han informado desde ninguna hasta el cinco por ciento.

Se ha descrito estenosis cervical en un tres por ciento (4), menos frecuente el mucometra como consecuencia de la estenosis del cuello (13). La reactivación de la enfermedad inflamatoria pélvica se puede presentar si esta ya existía y no fue detectada.

También se ha informado hemorragia vaginal, fenómenos vagales de choque, en las pacientes embarazadas amenaza de parto prematuro y ruptura de membranas.
Fracaso terapéutico o persistencia de la lesión es la confirmación histológica del NIC entre los cuatro y doce meses post-crioterapia.

Recurrencia o recidiva es la confirmación histológica del NIC después de doce meses post-tratamiento.

\section{Comentarios}

La crioterapia es una modalidad terapéutica conservadora, no requiere anestesia (32), no necesita hospitalización la paciente. Puede realizarse como un procedimiento de consultorio, y no requiere preparación por parte de la paciente, además, la fertilidad no se altera lo que la hace ideal para pacientes jóvenes con NIC III. Su costo es bajo comparado con los rayos láser (31) y no interfiere con las actividades diarias.

$\mathrm{Al}$ seleccionar este método como tratamiento, se debe encuadrar en un protocolo rígido de investigación que efectúe una selección estricta de las pacientes y tenga un seguimiento cuidadoso.

El siguiente flujograma da una idea del sitio que ocupa la crioterapia en el manejo del NIC.

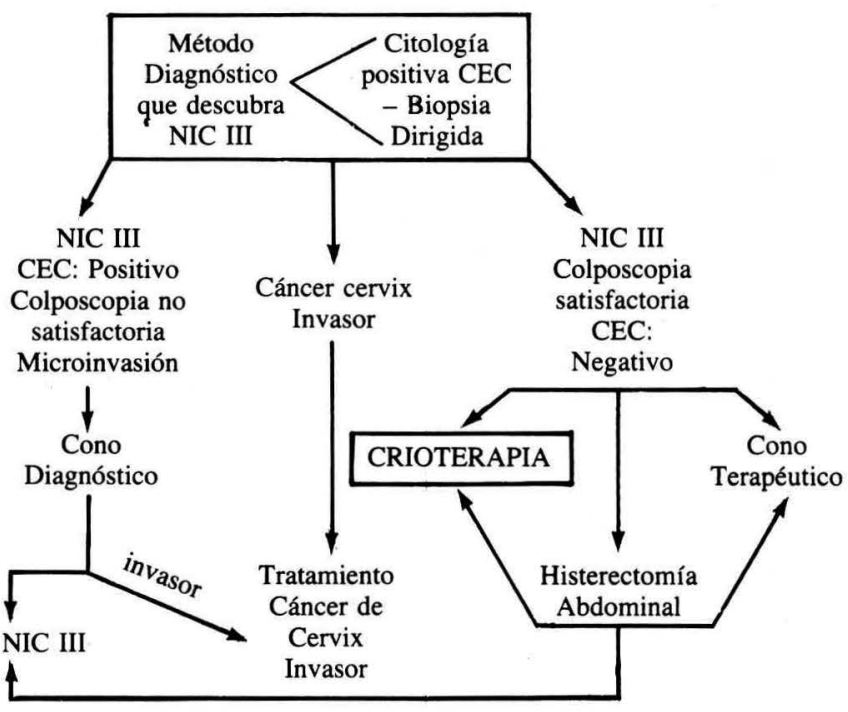

Es el método alternativo para el manejo de lesiones cervicales in situ, pero no se debe abusar de él porque si los casos no son seleccionados estrictamente, podemos enmascarar o pasar desapercibido el carcinoma microinvasor (25).

\section{BIBLIOGRAFIA}

1. Arof HM, Gerbic MV, Smeltzer J. "Cryosurgical treatment of cervical intraepithelial neoplasia: Four years experience”. Am J Obst Gynecol 1984; 150. (7): 865-9.

2. Anderson ES, Thorup K, Larsen G. "The results of cryosurgery for cervical intraepithelial neoplasia". Gin Oncol 1988; 30. (1): 21-25.

3. Botero J, Jubiz A, Henao G. "Texto Integrado Obstetricia y Ginecología", págs. 562-571, Medellín (Col.), Carvajal, 4ª ed., 1989.

4. Berget Ame, Lenstrup C. "Outpatient treatment of cervical intraepithelial neoplasia". Acta Obst Gyn Scond 1987; 66: 531-536.

5. Bryson SC, Lenehan P, Lickrish GM. "The treatment of grade 3 cervical intraepithelial neoplasia with cryotherapy an 11 years experience". Am J Obst Gynec 1985; 151. (2): 201-6.
6. Benedet JL, Miller DM, Nickerson KG. "The results of cryosurgical treatment of cervical intraepithelial neoplasia at one, five and ten years". Am J Obst Gyn Ang 1987; 157 (2).

7. Coney P, Walton LA, Edelman DA, Fowler WC. "Cryosurgical treatment of early cervical intraepithelial neoplasia". Obst Gynecol 1983; 62. (4): 463-6.

8. Creasman WT, Hinshaw WM, Clarke, Pearson DL. " Cryosurgery in the management of cervical intraepithelial neoplasia". Obst Gynecol 1984; 63. (2): 14509.

9. Diasia Philip. "Preinvasive disease of the cervix vaginal and vulva", Clinical Ginecologic Oncology. The C.U. Mosby Company, St Louis, Washington. 3th ed. 1989. 
10. Elsworth CH, Savage E. "Cryosurgical treatment of cervical intraepithelial neoplasia". Obst Gyn Survey 1980; 35. (9).

11. Einerth Y. "Cryosurgical treatment of NIC I - III a long term study". Acta Obst Gyn Scond 1988; 67. (7): 27- 30.

12. Ferenczy Alex. "Comparación de crioterapia y láser dióxido de carbono para la neoplasia intraepitelial cervical”. Obst Gynecol 1985; 66: 793-798.

13. Guison FB. "Mucometra a rare complication of cryosurgery". Am J Obst Gynecol 1988; 159. (1): 26.

14. Gallego G, Uribe J. "Análisis del NIC en el Hospital Universitario San Vicente de Paúl, 1970-1987”. Rev Col Obst Gin 1988; 39. (3)

15. Hans B Krebs, Whee Lock JB. "Raspado endocervical después de la crioterapia por una neoplasia intraepitelial cervical". J Repord Med 1985; 30: 379-382.

16. Hemmingsson E. "Cryosurgical treatment of cervical intraepithelial neoplasia with follow up of five to eight years". Am J Obst Gynecol 1981; 130. (2): 144-147.

17. Jones DE, Russo JF, Dombroski RA, Lenix SS. "Cervical intraepithelial neoplasia in adolescents". J Adolesce Health Care 1984; (4): 243-7.

18. Kirwan PH, Smith IR, Naftalin NJ. "A study of cryosurgery and the $\mathrm{CO}_{2}$ laser in treatment of carcinoma in situ (NIC III) of the uterine cervix". Gynecol Oncol 1985; 22(2): 195-200.

19. Kwikkel HJ, Helmerhorst TD. "Láser o crioterapia para la neoplasia epitelial cervical. Un estudio al azar para comparar la eficacia y efectos colaterales". Gynecol Oncol 1985; 22: 23-31.

20. Lent Van, Trimbos JB, Heintz PM. "Cryosurgical treatment of cervical intraepithelial neoplasia (NIC III) in 102 patients". Gin Oncol 1983; 16: 240-245.

21. Matsunaga Jon, Bergman A Bnatian. "Genital condylomata acuminata in pregnancy: Efectiveness, safety an pregnancy outcome following cryotherapy". Br J Obs Gin 1987; 94: 168.
22. Norum M, Dean L, Moyer MD, Towsend. "Ultraestructural changes in normal human ectocervical epithelium inmediately following cryosurgery". Lab Invest 1969; 21. (1).

23. Perdomo Rafael. "Tratamiento de las neoplasias intraepiteliales con criocirugía". Universidad Javeriana, Hospital San Ignacio, Departamento de Obstetricia y Ginecología, Santafé de Bogotá, D.C. 1980.

24. Peckham BM, Sonek M, Carr W. "Outpatient therapy: Success and failure with dysplasia and carcinoma in situ". Am J Obst Gyn 1982; 142(3): 323-329.

25. Sevin B, Ford I, Girtanner R, Hoskins W. "Invasive cancer of the cervix after cryosurgery". Obst Gyn 1979; 53(4).

26. Syrjanen K, Vayrynens, Saarikoski R. "Evolución natural de las infecciones cervicales por papiloma virus humano con base en un segmento prospectivo". Br J Obst Gin 1985; 92: 1086-1092.

27. Reyna Ramiro, Vargas Eleazar, Rivas Héctor. "Criocirugía en carcinoma intraepitelial del cervix”. Gin y Obst de México 1989; 57.

28. Towsend D, Richart R. "Cryotherapy and carbon dioxide laser management of cervical intraepithelial neoplasia: A controlled comparison". Obst Gyn 1983; 61(1): 75-78.

29. Gschnait F. "Human papilloma viruses". Tecnical Report No. 3. Viena, Austria, 1987.

30. Winceslans SJ. "Monaging women with human papilloma virus changs in cervical cystology, genitourin". Med (England) 1988; 64(4): 259-262.

31. Wetchler SJ. Treatment of cervical intraepithelial neoplasia with the $\mathrm{CO}_{2}$ Laser: laser versus cryotherapy. A review of effectiveness and cost". Obst Gyn Survey 1984; 39(8): 464-73.

32. Zaninitti P, Franceschi S, Ballolo M, Bonazzi B. "Caracteristic of women under 20 with cervical intraepithelial neoplasia". Inter $\mathbf{J}$ of Epidemiology 1986; 15(4). 